\title{
Research Status and the Problems of Traditional Chinese Medicine Sterilization
}

\author{
Shaozhen Chen \\ Guangdong Lingnan Institute of Technology, Guangzhou, Guangdong, China
}

Keywords: Traditional Chinese medicine, Sterilization process, Current situation, Existing problems, Future prospects

\begin{abstract}
In the quality control of traditional Chinese medicine and its preparations, the sterilization process is an important operation unit, which has an important impact on the safety, effectiveness and quality stability of pharmaceutical products. Therefore, in the production of modern Chinese medicine preparations, it is necessary to fully master the sterilization process. In view of this, this article mainly analyses the current status of the use of traditional Chinese medicine sterilization technology, and proposes relevant effective measures for its existing problems, points out the direction for the future development of traditional Chinese medicine sterilization technology, and promotes the development and development of new sterilization technologies for traditional Chinese medicine and its preparations. The conversion level is further improved.
\end{abstract}

\section{Introduction}

At this stage, countries around the world have gradually begun to pay attention to the study of drug microbial limits to ensure the safety, effectiveness and quality stability of traditional Chinese medicines and their preparations. In response to this, my country has made detailed regulations on its sterilization operations in the new version of the "Pharmaceutical Production Quality Management Code”. Therefore, higher requirements are put forward for the operation of the sterilization process of traditional Chinese medicine, and the problems existing in the implementation of the actual sterilization process are analysed and explored, and relevant effective methods are proposed to ensure the safety of the use of traditional Chinese medicine and its preparations, improve the efficacy, and follow-up traditional Chinese medicine The development of sterilization technology has laid a good foundation to accelerate the development of traditional Chinese medicine and preparation sterilization technology.

\section{Current Status of Chinese Medicine Sterilization Process}

\subsection{Physical Sterilization Process}

The physical sterilization process of traditional Chinese medicine and preparations mainly includes heat sterilization and radiation sterilization, and heat sterilization includes moist heat sterilization and dry heat sterilization. The specific status is as follows:

Moisture heat sterilization is a traditional method that is currently widely used, and is considered to be the most convenient, most reliable, and effective sterilization technology without harmful residues. In actual operation, according to the difference of temperature and pressure, moist heat sterilization technology can be divided into high pressure sterilization technology, circulating steam sterilization technology, low temperature intermittent sterilization technology and superheated water spray sterilization technology. Different sterilization techniques can be used to obtain different sterilization effects, but the principle is mostly to use the protein, nucleic acid and other substances contained in the microbial cells to effectively kill harmful microorganisms after denaturation and solidification. Moreover, the moist heat sterilization process is suitable for the sterilization operation of various solid, semi-solid and liquid preparations, and the sterilization technology is reasonably selected according to the nature of the sterilized drugs. For example, for traditional Chinese medicine oral liquids or syrup preparations, the steam sterilization method can be used, and the superheated water spray method should be used to sterilize drugs such as injections 
or large infusions. The current application of moist heat sterilization process has the advantages of better actual sterilization effect, simple and reliable equipment, and relatively low cost. However, for important components with volatility and easy oxidation characteristics, steam will cause degradation and destruction. Phenomenon, it is easy to cause volatilization loss, reduce curative effect, and even produce certain toxic side effects. Therefore, when using the moist heat sterilization method, the ingredients of the drug should be fully considered to prevent its quality and drug safety from being affected [1].

Dry heat sterilization refers to the use of an electric thermostat or a heating device inside a hard air duct to raise the temperature to a certain level to kill microorganisms within a specified time. The main principle is to use high-temperature dehydration and drying to oxidize proteins, and then after denaturation, carbonization and electrolyte concentration poisoning, the microorganisms can be killed. It is usually more suitable for the sterilization of raw and auxiliary materials such as talcum powder, Chinese herbal medicine powder and low-moisture ointment. When carrying out the dry heat sterilization method, it is necessary to ensure that the temperature is $160-170^{\circ} \mathrm{C}$ and the sterilization time is specified above $2 \mathrm{~h}$, or when the temperature is $250^{\circ} \mathrm{C}$, the sterilization time is specified above $45 \mathrm{~min}$. The more common dry heat sterilization method is tunnel type, which has better sterilization effect, less moisture content, and avoids problems such as the occurrence of powder sterilization and compaction. However, in the implementation, due to the relatively high temperature of the dry heat sterilization method and the long sterilization time, it may cause the color of some virgin drug powders to darken and cause the volatile components to escape. Therefore, for those containing volatile Dry heat sterilization is not suitable for the sterilization of traditional Chinese medicines with high oil content, sugars, fats, etc. [2].

For the radiation sterilization of Chinese medicines and preparations, ionizing radiation and electromagnetic wave radiation are mainly used. The radiation source of ionizing radiation is generally 60Co. The principle of sterilization is that after the microorganisms are irradiated by the radiation, it will have two effects, direct and indirect. The direct effect means that the cell nucleus will be hit by the radiation and die. The indirect effect is due to the cell nucleus. It contains a lot of water. When the water fully absorbs the radiant energy, it will generate more active particles. When it interacts with proteins and enzymes, it will change the structure or material needed for life, causing it to die. The main advantage of this kind of sterilization process is that it can be sterilized at room temperature, the whole irradiation process hardly rises, and the sterilization time is relatively short, often only a few seconds. It has a good sterilization effect on Chinese medicinal materials that contain volatile components and heat-sensitive components, high sugar content, and prone to mildew.

Most of the electromagnetic wave radiation sterilization uses microwave, the principle of which has thermal effect sterilization and non-thermal effect sterilization. First, microwave high frequency can use alternating electric field to promote the concentration and dissolution of nuclei due to heating of bacteria, thereby achieving sterilization; secondly, microwave In the electromagnetic field, the bacteria will produce a relatively strong reaction, which can effectively penetrate the cell membrane of the capacitive structure to kill the bacteria. It is suitable for liquid medicine, pills, tablets and other sterilization processes.

\subsection{Chemical Sterilization Process}

The chemical sterilization process for traditional Chinese medicines and preparations includes ethanol steam sterilization technology and ozone sterilization technology.

Ethanol steam sterilization is to mix $70 \%$ ethanol into the ethanol steam with high volume fraction and keep its volume fraction at about 75\%, which can form a good sterilization concentration, at a temperature of about $65^{\circ} \mathrm{C}$ In the environment, airtight vacuum, maintain it at $0.08 \mathrm{MPa}$, after 8 hours of cooling, the bacteria can be eliminated, and the sterilization process can be repeated. In the process of carrying out the sterilization process, it should be noted that some Chinese medicines are sterilized by high temperature after adding an appropriate amount of ethanol. The essence is close to the "wine moisturizing" method of the Chinese medicine processing process. 
Therefore, it will have a greater impact on the medicinal properties of Chinese medicine, so when using it, attention should be paid to the medicinal properties and ingredients of the medicinal materials.

Ozone sterilization is a method that has a relatively strong killing effect on bacteria, molds and viruses. This is due to the strong oxidizing ability of ozone itself. After its disinfection and sterilization process is completed, it can self-decompose and reduce to oxygen without producing any toxic and harmful substances. It is a safe, efficient, and new new sterilization technology without residual pollution. The principle uses the instability of ozone, which releases free radical oxygen during decomposition, which can penetrate the cell wall, oxidize the glucose in the bacteria into enzymes, destroy the RNA or DNA substances of the bacteria and viruses, in order to achieve the killing of bacteria [3].

\subsection{New Sterilization Process}

Modern new sterilization processes mainly include high-pressure pulse power plant sterilization, low-temperature plasma sterilization, and instantaneous ultra-high-pressure sterilization at room temperature. The principle of the high-voltage pulsed electric field sterilization method is to rely on electric disintegration and electroporation to promote the gradual rupture of the cell membrane through the external electric field, thereby improving the disorder and permeability of the cell membrane structure, resulting in the loss of the vitality of various biological enzymes. Eventually cause cell death. Electroporation uses high-voltage electrical pulses to change the structure of fat molecules and enlarge the protein channels. When a large amount of current passes through, the cells swell and die. It has the advantages of short process time, low temperature rise, high sterilization efficiency, and does not affect the composition of the drug; low-temperature plasma sterilization generally works under the action of heating or strong electromagnetic fields, and is composed of electrons, ions, and atoms. It is composed of molecules, active free radicals, rays, etc. It is a new form of aggregation of substances in addition to solid, liquid and gaseous states. Its advantages are lower sterilization temperature, fast speed, no toxic residues, higher sterilization effectiveness, etc., especially for heat-sensitive medicinal materials; the instantaneous ultra-high pressure sterilization method at room temperature refers to the ultra-high pressure sterilization Under conditions, the protein maintains a spherical state, reduces the distance between water molecules, and penetrates into the vicinity of amino acids inside the protein. Once the ultra-high pressure occurs and instantaneous decompression occurs, water molecules will vaporize and explode, destroying the spatial structure of the protein, in order to achieve the purpose of sterilization. This process method is more suitable for traditional Chinese medicine preparations containing heat-sensitive and volatile components, and has the advantages of high efficiency, safety, and pollution-free [4].

\section{Current Problems in the Sterilization Process of Traditional Chinese Medicine}

Although the current Chinese medicine sterilization technology has very good development, it still has certain problems. Moreover, different sterilization technologies will have a greater impact on the quality of traditional Chinese medicines. Therefore, in the actual sterilization process, a reasonable selection of sterilization methods should be based on the nature and characteristics of Chinese medicines and preparations. In the process of using traditional dry heat sterilization, moist heat sterilization, and chemical sterilization, the active ingredients of traditional Chinese medicine will be destroyed to a large extent, and some toxic substances may even remain. As a result, the sterilization of traditional Chinese medicines and preparations has large quality problems, which is not conducive to the safety and effectiveness of the medication. Therefore, effective strategies and methods must be adopted in the sterilization process to promote the improvement of sterilization problems and protect traditional Chinese medicines and preparations. The quality stability. 


\section{Strategies and Methods of Traditional Chinese Medicine Sterilization Process}

\subsection{Avoid Destruction and Degradation of Active Ingredients}

Due to the relatively complex chemical components of traditional Chinese medicines and preparations, in the sterilization process, the heat-sensitive active ingredients contained in some traditional Chinese medicines will be destroyed under the influence of high temperature, resulting in volatilization loss and degradation, which will have an adverse effect on the therapeutic effect of the medication. , Serious may cause adverse reactions, which is not conducive to medication safety. Therefore, in the sterilization process of traditional Chinese medicine and preparations, it is necessary to adopt appropriate sterilization technology according to the characteristics of the medicine to avoid the destruction and degradation of the effective components of the traditional Chinese medicine to the greatest extent, so as to ensure the safety and effectiveness of the medicine.

\subsection{Elimination of Hazardous Residues}

In the important sterilization process, under the action of different technical methods, certain chemical reactions may occur to generate toxic and harmful substances. If it remains in medicines, it will pose a huge safety threat to users. Therefore, when using radiation sterilization, it is necessary to strictly examine the nature of traditional Chinese medicines, use advanced detection technology to avoid harmful residual substances, and ensure the safety of traditional Chinese medicines as much as possible. On the other hand, when the sterilization process is carried out, new technologies should be actively adopted, such as high-pressure pulse power plant sterilization, lowtemperature plasma sterilization, and instantaneous ultra-high-pressure sterilization at room temperature, which will not produce pollutants and have better The safety of [5].

\section{Prospects for the Future Development of Chinese Medicine Sterilization Technology}

Sterilization process is one of the indispensable key links in the pharmaceutical process of traditional Chinese medicine, which will have a greater impact on the quality of medicines. At the same time, in the entire Chinese medicine pharmaceutical industry, the research and development of sterilization technology is also an important manifestation of its modernization transformation. Therefore, in the future development process, the traditional Chinese medicine sterilization process will develop in the direction of technical standardization, high efficiency, and low-carbon upgrade. The specific development trends are as follows:

(1) Pay attention to the special advantages of various sterilization technologies, realize the combination of two or more sterilization technologies, and promote the development of the sterilization process towards integration and modularization.

(2) Strengthen basic research on traditional Chinese medicine and preparations, such as theoretical analysis of adaptability, sterilization mechanism, sterilization equipment, etc., so as to guide the effective development and improvement of sterilization treatment processes.

(3) Increase national policy support. Based on the development situation of the traditional Chinese medicine pharmaceutical industry, my country's national policy will focus on the industrial application of traditional Chinese medicine sterilization technology, and vigorously support and guide the continuous innovation and advancement of sterilization technology.

(4) Sterilization technology research and development Strengthen exchanges and cooperation with enterprises and the front line of production to ensure that the process technology aims at the actual development needs of enterprises, develop personalized Chinese medicine and preparation sterilization technology, and fully solve the actual problems that occur in the sterilization process.

\section{Conclusion}

In summary, the traditional Chinese medicine sterilization process is an important link and unit in the pharmaceutical industry at this stage, and it has a key impact on the safety, effectiveness, and 
quality stability of traditional Chinese medicine and preparations. Therefore, in order to ensure the quality of medicines, it is necessary to reasonably select an appropriate sterilization process according to the properties of Chinese medicinal materials. And in the actual implementation process, it effectively avoids the destruction and degradation of the active ingredients of the medicinal materials and eliminates the residue of harmful substances, thereby improving the production quality of traditional Chinese medicines and preparations, and exerting their due therapeutic effects.

\section{References}

[1] Lin Tong, Bi Fujun, Lu Weisheng, Zhang Liwen, Jin Hongyu, Ma Shuangcheng, Jiang Yingqiao. Current status and supervision of irradiation sterilization of traditional Chinese medicine[J]. Chinese Pharmaceutical Journal, vol. 54, no. 17, pp. 1442-1447, 2019.

[2] Wei Yulong, Lan Xiaoqing. Research progress in the application of traditional Chinese medicine cobalt 60 irradiation sterilization. Strait Pharmacy, vol. 31, no. 8, pp. 4-7, 2019.

[3] Sun Yu. Discussion on sterilization methods of traditional Chinese medicine. Chinese Journal of Clinical Pharmacology, vol. 34, no. 19, pp. 2380-2382, 2018.

[4] Zhao Wenjia, Zhao Xin, Jin Hongfei, Wu Pinchang. Experimental study on the sterilization of traditional Chinese medicine disinfection emulsion. Journal of Practical Traditional Chinese Medicine, vol. 34, no. 4, pp. 389-390, 2018.

[5] Wang Shuang, Nie Qixia, Zhang Baoxian, Zhang Liyan, Zang Chen, Wang Guohua, Shang Yue. Overview of the application of microwave drying and sterilization technology in the field of traditional Chinese medicine. China Journal of Information on Traditional Chinese Medicine, vol. 24, no. 11, pp.132- 136, 2017. 